\title{
Effect Analysis of Opening Wedge High Tibial Osteotomy (OWHTO) for the Treatment of Medial Compartment Osteoarthritis
}

\author{
Songwei Huan, Ning Liu, Simin Luo, Huige Hou, Guorong She, Tengfeng Zhuang, Zhengang Zha* \\ Department of Orthopedics, The First Affiliated Hospital, Jinan University, Guangzhou, China \\ Email address: \\ jnuhuan@163.com (Zhengang Zha) \\ ${ }^{*}$ Corresponding author \\ To cite this article: \\ Songwei Huan, Ning Liu, Simin Luo, Huige Hou, Guorong She, Tengfeng Zhuang, Zhengang Zha. Effect Analysis of Opening Wedge High \\ Tibial Osteotomy (OWHTO) for the Treatment of Medial Compartment Osteoarthritis. Journal of Surgery. Vol. 7, No. 3, 2019 , pp. 57-62. \\ doi: $10.11648 /$ j.js.20190703.12
}

Received: April 14, 2019; Accepted: May 21, 2019; Published: May 30, 2019

\begin{abstract}
To investigate the clinical efficacy of open wedge high tibial osteotomy (OWHTO) in the treatment of medial compartment osteoarthritis of the knee. The authors used open OWHTO combined with TomoFix internal fixation to treat 46 cases (52 knees) with knee osteoarthritis with patella varus deformity from 2015.01 to 2017.10. HSS score and VAS score of Knee joint were used to evaluate the improvement of knee function and pain before and after surgery. The femorotibial angle (FTA), medial proximal tibial angle (MPTA), kneecap varus angle (KVA), and Weight-bearing line ratio of lower limbs (WBL) were measured by videography, which can evaluate the correction effect of postoperative knee varus. There were no happenings of internal fixation failure, delayed bone healing or non-union in these follow-up patients. At the follow-up of 3 months, 6 months, and 12 months, the HSS scores of the patients were significantly higher than those before the operation, and the difference was statistically significant $(\mathrm{P}<0.05)$. On March, June, and December after surgery, the HSS scores of patients were significantly higher than those before surgery and the difference was statistically significant $(\mathrm{P}<0.05)$. The VAS scores were lower than those before surgery, and the difference was statistically significant $(\mathrm{P}<0.05)$. These scores had no significant difference in the different follow-up time points ( $\mathrm{P}>0.05)$. Imaging examination indexes showed that FTA, MPTA, KVA and WBL were improved after surgery and the varus deformity was corrected. Moreover, the difference was statistically significant $(\mathrm{P}<0.05)$. These scores had no significant difference in the different follow-up time points $(\mathrm{P}>0.05)$. In general, OWHTO can relieve joint pain and improve joint function by correcting lower limb line, showing good effect on medial compartment osteoarthritis of knee joint with varus.
\end{abstract}

Keywords: High Tibial Osteotomy, Open Wedge High Tibial Osteotomy, Knee Varus Deformity, Osteoarthritis, Lower Extremity Line

\section{Introduction}

As early as 1958, Jackson et al [1] reported that the osteotomy and correction of upper tibia can be used to treat knee osteoarthritis with varus. This method was improved by Coventry in 1965 and he reported that wedge osteotomy above the level of the tibial tuberosity can treat medial single compartment osteoarthritis with a certain degree of knee varus to a certain extent. This method had achieved good long-term efficacy, so it was named High tibia osteotomy (HTO) and gradually promoted. In the promotion and application, there were two different osteotomy methods: lateral closed type and medial open type. Since there are many complications such as limb shortening, tibia osteotomy, and common peroneal nerve injury in the lateral closed type [2-5]. Therefore, open wedge high tibia osteotomy (OWHTOW) is currently used in clinical practice. With the promotion of surgical techniques and the development of internal fixation materials, OWHTO has attracted more and more scholars' attention in the treatment of "knee-protection" of relatively young patients. The author used OWHTO combined with TomoFix internal fixation to treat the medial compartment osteoarthritis of the knee joint 
and patella varus. The author treated 46 patients ( 52 knees) from 2015.01-2017.10 and analyzed the clinical efficacy of OWHTO.

\section{Materials and Methods}

\subsection{General Information}

46 patients (52 knees) who underwent OWHTO surgery for the treatment of the medial compartment osteoarthritis of the knee joint in the First Affiliated Hospital of Jinan University from 2015.01 to 2017.10 , including 14 males (16 knees) and female 32 (36 knees); age $47 \sim 64(57 \pm 6)$ years, disease course of 2-13 years, an average of 6.5 years.

\subsection{Indications and Contraindications Indications}

(1) Medial compartment osteoarthritis of knee joint, (2) combined with humeral varus deformity, medial proximal tibial angle (MPTA) $<85^{\circ}$, kneecap varus angle (KVA) $>5^{\circ}$, (3) knee joint activity is basically normal, flexion deformity $<$ $10^{\circ}$, (4) The lateral compartment structure and the patellofemoral joint were basically normal, and there was no pain in lateral compartment structure and the patellofemoral joint before the surgery. Contraindications: (1) inflammatory, infectious arthritis, (2) severe or multi-ventricular joint degeneration, (3) knee joint mobility $<90^{\circ}$, fixed flexion deformity $>10^{\circ}$, (4) obvious joint instability, 5BMI $>30$.

\subsection{Preoperative Planning}

Full-length positive $x$-ray film in standing position of both lower limbs was shot before the surgery. It should be noted that the patient was required to have a 15 degrees of foot pronation, the humerus was located directly in front of X-ray film, and one third of the inside caput fibulae overlaped with the proximal tibia. MPTA was measured and the angle of osteotomy correction which was required by coronal plane was also measured according to Miniaci [6] force line method. The corrected negative gravity line of the tibial plateau is $62.5 \%$ from the inside to the outside, that is, Fujisaw point [7].

\subsection{Surgical Method}

After the combined spinal and epidural anesthesia, A short oblique incision was made on the medial side of the humerus, which started from 1 transverse finger below medial articular line and extended to the lower part of the tibial tuberosity, about $7 \mathrm{~cm}$. Skin, subcutaneous and fascia were cut open, and adipose tissue was stripped. Then the goose foot and periosteum were partially cut about $3 \mathrm{~cm}$ below the joint line. The medial and posterior tibia were stripped and exposed by strict subperiosteal, which could prevent the damage of the posterior blood vessels. Two Kirschner wires were drilled in parallel to the direction of the tibia cap about $4 \mathrm{~cm}$ from the inside to the outside of the tibial plateau. We should ensure that the tip of the kirschner wire was located $1.0 \sim 1.5 \mathrm{~cm}$ below the outer platform under fluoroscopy. Osteotomy plane was marked by the means of biplane osteotomy. The first osteotomy surface is the plane where the Kirschner wire is located and it obliques to the lateral tibia from inside to outside. So, attention should be paid to the depth of osteotomy, and the lateral cortex of the tibia should be kept at $1.0 \mathrm{~cm}$ to ensure the continuity of the lateral cortex. At the same time, what need to aware of is protecting the vascular nerve behind the humerus. The second osteotomy surface is located at the posterior inferior tibial tuberosity from the distal end to the proximal end and it is at an angle of about $110^{\circ}$ with the first osteotomy surface. Attention should be paid to be careful not to damage the patellar ligament. Then several bone knives and angle spreaders were used to gradually open the angle to the angle to be corrected, moreover, the opening process must be gentle and slow. For younger patients with better bone quality, the fine Kirschner wire can be used to perforate the lateral cortex before the opening, which can weak the hinge and prevent fracture of the lateral cortex when distracting. After fluoroscopy confirming that the force line is corrected, the opening angle was maintained and the TomoFix locking plate was placed on the inside of the proximal humerus. Attention should be paid to the height of the placement, preventing the proximal screw penetrating into the joint, which will damage the cartilage. The screws are locked in sequence, and the first screw of the distal end is firstly pressurized and then replaced with a locking nail to enhance the stability of the internal fixation. If the correction angle is less than $15^{\circ}$, bone grafting will not be required at the osteotomy site. If the correction angle is greater than $15^{\circ}$ or the patients have osteoporosis, obesity, artificial bone or iliac bone should be grafted, which can prevent complications such as delayed healing or non-union of the osteotomy, loss of correction angle or failure of internal fixation. The wound should be rinsed repeatedly and sutured to the skin layer by layer.

\subsection{Postoperative Treatment}

Multimodal analgesia was performed after operation and sputum pump movement and passive knee movement were started after anesthesia resuscitation, which could prevent of deep venous thrombosis of lower limbs. The rehabilitation training was started with the aid of a walker in 2-3 days after surgery, and the load was about $15 \sim 20 \mathrm{~kg}$. With the degree of pain gradually increasing, X-ray examination was implemented in 6 weeks after surgery to confirm that there was a bone healing sign on the lateral side of the osteotomy and the upper osteotomy surface, and the full weight bearing can be started.

\subsection{Efficacy Evaluation}

Follow-up visit was performed at 3, 6, and 12 months after surgery. Knee joint function was assessed by the score of the American Special Surgery Hospital (HSS) and pain level was assessed by the score of the Pain Visual Analog Scale (VAS). At the follow-up, the full length X-ray of the lower extremities were reviewed, so the effect of proximal varus correction could be evaluated from several aspects: femorotibial angle 
(FTA), medial proximal tibial angle (MPTA), kneecap varus angle (KVA), and Weight-bearing line ratio of lower limbs (WBL).

\subsection{Statistical Analysis}

SPSS 19.0 was used to perform statistical analysis and the measurement data were expressed as mean \pm standard deviation. The multiple time points before and after surgery were compared by repeated measures analysis of variance and LSD test was used to compare the two pairs $\alpha=0.05$.
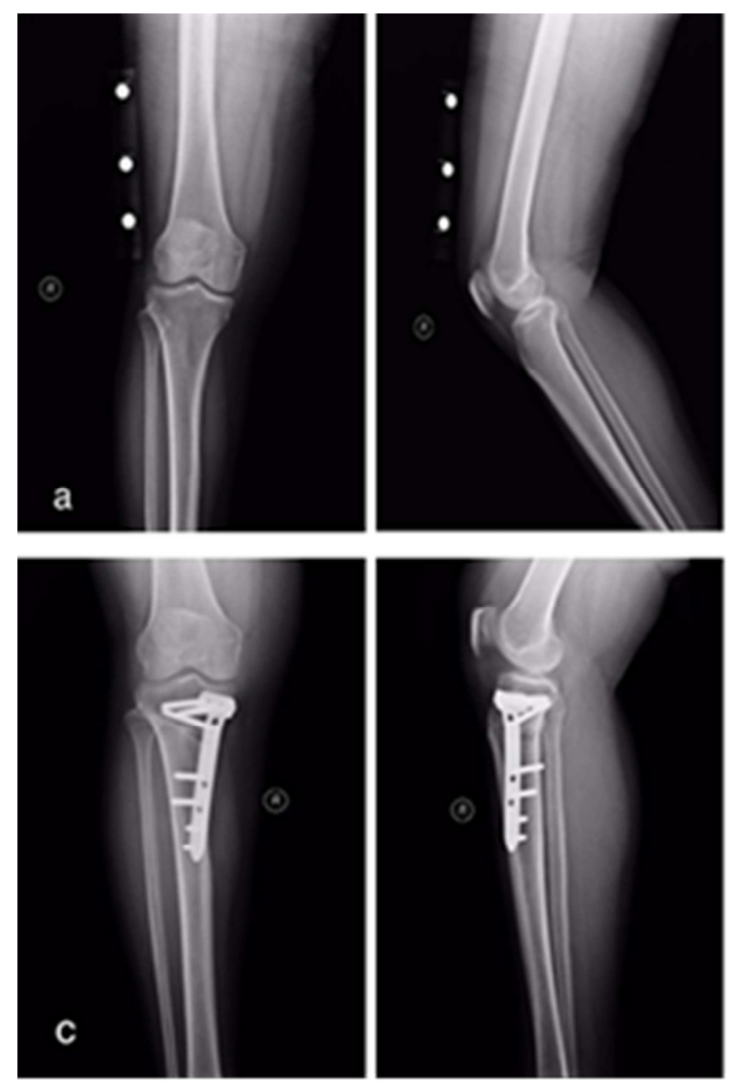

\section{Results}

All the postoperative incisions were healed by the first stage. 3 cases of the patients had no displacement fracture of osteotomy axis of rotation, which delayed healing after loading. 4 cases of the patients had postoperative calf swelling and subcutaneous ecchymosis, which were eased after symptomatic treatment. All patients were randomly visited for 13 to 28 months with an average of 18.2 months. There were no happenings of internal fixation failure, delayed bone healing or non-union in these follow-up patients.

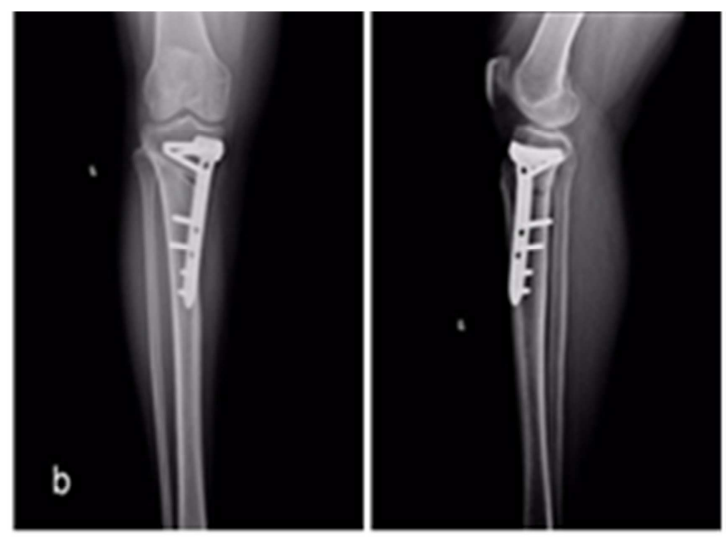

Figure 1. The patient who was female and 57 years old, the X-ray film of right knee joint medial compartment arthritis anteroposterior a. preoperative; $b .3$ months after surgery, the outer edge of the osteotomy line was blurred, osteophyte formation; c. After 12 months, the most part of osteotomy gap had healed.

The knee function of the patients was significantly improved after surgery, and the pain was relieved. The HSS score and VAS score at each follow-up point were significantly different from those before surgery $(\mathrm{P}<0.05)$ and there was no significant difference between the time points $(\mathrm{P}>0.05)$. (Table 1)

Table 1. Comparison of VAS and HSS scores between pre- and post-operation $(n=52)$.

\begin{tabular}{|c|c|c|c|c|c|c|c|c|}
\hline \multirow[b]{2}{*}{ time } & \multirow[b]{2}{*}{ VAS score } & \multicolumn{7}{|l|}{ HSS score } \\
\hline & & pain & function & activity & $\begin{array}{l}\text { Muscle } \\
\text { strength }\end{array}$ & $\begin{array}{l}\text { Flexion } \\
\text { deformity }\end{array}$ & stability & Total score \\
\hline Preoperation & $6.92 \pm 1.29$ & $12.51 \pm 1.45$ & $12.71 \pm 3.71$ & $13.02 \pm 2.01$ & $7.13 \pm 2.45$ & $5.17 \pm 0.67$ & $5.01 \pm 0.57$ & $60.25 \pm 5.88$ \\
\hline Post-3 months & $3.84 \pm 2.07$ & $21.12 \pm 2.64$ & $16.97 \pm 2.01$ & $15.89 \pm 5.13$ & $8.51 \pm 1.87$ & $8.70 \pm 0.84$ & $7.98 \pm 0.48$ & $82.11 \pm 6.01$ \\
\hline Post-3 months & $3.12 \pm 2.41$ & $23.12 \pm 1.78$ & $18.17 \pm 2.27$ & $16.33 \pm 2.87$ & $8.98 \pm 1.44$ & $8.87 \pm 0.52$ & $8.18 \pm 0.70$ & $84.56 \pm 6.27$ \\
\hline Post-3 months & $2.56 \pm 1.47$ & $23.49 \pm 1.11$ & $18.32 \pm 1.97$ & $16.55 \pm 1.54$ & $8.76 \pm 1.10$ & $8.90 \pm 0.69$ & $8.17 \pm 0.64$ & $85.33 \pm 5.73$ \\
\hline F value & 176.22 & 201.32 & 185.22 & 352.99 & 289.10 & 197.33 & 122.77 & 369.47 \\
\hline
\end{tabular}

Imaging examination indexes showed that FTA, MPTA, KVA and WBL were improved after surgery and the varus deformity was corrected, and the lower limb negative gravity line was adjusted to slight eversion position (Fujisawa point). Moreover, the difference was statistically significant $(\mathrm{P}<0.05)$. These scores had no significant difference in the different follow-up time points $(\mathrm{P}>0.05)$. (Table 2) 
Table 2. Comparison of radiological parameters between pre-and post-operation $(n=52)$.

\begin{tabular}{llll}
\hline time & PTA $\left({ }^{\circ}\right)$ & MPTA $\left({ }^{\circ}\right)$ & KVA $\left({ }^{\circ}\right)$ \\
\hline Preoperation & $186.87 \pm 4.55$ & $81.71 \pm 2.14$ & $10.17 \pm 1.87$ \\
Post-3 months & $171.25 \pm 5.22$ & $95.47 \pm 1.88$ & $1.97 \pm 2.18$ \\
Post- 6 months & $171.85 \pm 3.81$ & $95.02 \pm 1.47$ & $2.12 \pm 1.37$ \\
Post-12 months & $171.46 \pm 2.87$ & $94.10 \pm 1.79$ & $2.60 \pm 2.00$ \\
F value & 287.41 & 177.96 & 461.33 \\
P value & 0.00 & 0.00 & 0.00 \\
\hline
\end{tabular}

\section{Discussion}

Knee osteoarthritis is a kind of common chronic disease and frequently-occurring disease, which is usually occurred in middle-aged and elderly people. It usually occurs in cartilage and is accompanied by multiple systemic diseases such as synovium, meniscus and ligaments around the joints, which eventually leads to joint swelling, pain, and deformity. This disease can even lead to some patients being unable to take care of themselves. Joint replacement can be used to correct deformities, improve activity, and restore the physiological function of the knee joint for the patients who have end-stage knee osteoarthritis. However, for the patients who have middle and early knee osteoarthritis, the degree of lesions is relatively low and the extent of lesions is often limited, so artificial joint replacement is too early to be used on these patients. In addition, these patients are often young and have higher requirements for quality of life and joint function [12-14]. Therefore, the High Tibial Oteotomy (HTO) which is confirmed the concept of "knee-protection" has been paid more and more attention by scholars in recent years. The basic idea of HTO is to correct the sacral varus deformity and appropriately remove the negative gravity line of the lower limb from the degenerating medial compartment, thereby the pressure of the medial compartment can be reduced. Moreover, the cartilage can be effectively prevented from being worn and degenerated and the pain can be relieved, which provide a self-healing environment for the worn medial cartilage and meniscus. The choice of indications for HTO is very important Postoperative residual pain or premature transitional joint replacement is usually associated with inappropriate selection of indications.

The best indications for HTO [12, 14-17] are: (1) The patients'age are less than 65 years old (female is less than 60 years old); (2) combined with humeral varus deformity, medial proximal tibial angle (MPTA) $<85^{\circ}$, kneecap varus angle (KVA) $>5^{\circ}$ (3) knee joint activity is basically normal, flexion deformity should be less than $10^{\circ}$ and force line deformity is less than $15^{\circ}$; (4) lateral cartilage and meniscus function are normal. HTO is an extra-articular surgery that can correct stress concentration in the joint and relieve pain by correcting patella varus outside the joint. Therefore, HTO is not effective for medial single-chamber osteoarthritis caused by non-tibia varus. whether the patella varus is merged can be measured by the X-ray of the lower extremity, which can show MPTA, distal distal angle of the femur and angle of joint line. In addition, the patient'the body should be carefully examined before surgery and lesions and pain sites should be confined on the medial side. Since combined lateral cartilage wear or lateral meniscus injury cannot be alleviated by HTO, and HTO may even aggravate this symptom after force line migration [15].

HTO is a kind of osteotomy orthopedic surgery and the key to its success lies in precise surgical techniques. OWHTO is a diagonal osteotomy on the inside of the humerus, which is gradually opened by the outer cortex as the axis of rotation, forming a wedge-shaped gap on the inner side. Therefore, it is especially important to maintain the integrity of the lateral cortex during surgery and the fracture of the lateral cortex is an important factor that lead to the early failure after HTO [8, 9]. A study by Kuremsky [10] showed that lateral cortical fractures would at least result in a loss of corrected angle of $4^{\circ}$ after surgery and an effective way to prevent lateral cortical fractures is to place the outer rotating shaft in the proper position. This position is located between the tip of the humeral head and the residual humeral head of the humeral head, moreover the position is $10-15 \mathrm{~cm}$ [11] away from the lateral edge of the tibial plateau.

In addition, gentle and slow expansion during operation is also an effective way to prevent fracture of the lateral cortex $[12,13]$. For young patients, the lateral cortex is hard, which can be punched with $1.5 \mathrm{~mm}$ Kirschner wire. In this way, the lateral cortex is moderately weakened, and the fracture of the lateral cortex can be prevented when distracted. In this group of patients, 3 cases of them had lateral cortical fractures. In these three cases, 1 case had low rotation axis position, 2 cases suffered from inadvertent intraoperative operation. However, the 3 cases all had no displacement fracture and all patients healed after delayed weight bearing.

OWHTO has a positive clinical effect on the treatment of medial compartment osteoarthritis with patella varus. For example, Hantes [14] used OWHTO to treat 20 cases of medial compartment osteoarthritis with patella varus, and the knee joint function was improved after surgery. The research of Al-Ghandour [14] shows that OWHTO is more suitable for young patients and they can have slight vigorous activities after 6 months. However, OWHTO still has some shortcomings and controversies in clinical applications. The first shortcoming is that OWHTO has a risk of affecting the height of the tibia. OWHTO osteotomy was performed above the tibial tubercle. Postoperative tibial tubercle shift towards the far end and the surface of the patellar ligament may be damaged by upper osteotomy, which may cause contraction of the patellar tendon and result in patellofemoral hypertension and even low tibia. Krause et al [14] have reported that subtuberosity osteotomy of tibia can effectively avoid low tibia and tibiofemoral hypertension, but it is easy to cause 
complications such as tibial tuberosity fracture. Moreover, OWHTO can cause tibial retroversion after surgery $[15,16]$. The slight increase in tibial retroversion will not have serious consequences, but the excessive tibial retroversion will increase the tension of the cruciate ligament, which can affect the joint activity, and increase the difficulty in changing the joint replacement in the future. Jacobi et al [17] found that the change of the tibial retroversion angle after OWHTO was related to the preoperative anatomy of the proximal humerus, the placement of the plate, the position of the lateral axis of rotation, and the lack of osteotomy of the posterior tibial cortex. At last, if OWHTO needs bone grafting is also controversial. Darees [18] believes that if TomoFix is used as internal fixation, knee varus does not require bone grafting within $15^{\circ}$, and most of the osteotomy sites can heal by themselves within 1 year. It is currently believed that bone grafting is related to the patient's bone quality, correction angle and internal fixation type [19-21]. In this group of studies, the patients had good bone quality and their distraction distance was less than $15 \mathrm{~mm}$. They all had no bone grafting and complications such as internal fixation failure and osteotomy and non-union.

\section{Conclusion}

OWHTO has a good clinical effect in the treatment of medial compartment osteoarthritis of knee with tibial varus. The operation is relatively simple, safe, and has few complications, so it is worthy of being promoted in some relatively young patients with knee osteoarthritis with a certain degree of patella varus. However, the number of cases in this study is limited, and the follow-up time is short. So, the long-term efficacy remains to be further observed.

\section{References}

[1] Jackson JP and Waugh W. Tibial osteotomy for osteoarthritis of the knee. J Bone Joint Surg Br 1961; 43-b: 746-751.

[2] van Egmond N, Stolwijk N, van Heerwaarden R, van Kampen A and Keijsers NLW. Gait analysis before and after corrective osteotomy in patients with knee osteoarthritis and a valgus deformity. Knee Surg Sports Traumatol Arthrosc 2017; 25: 2904-2913.

[3] Collins B, Getgood A, Alomar AZ, Giffin JR, Willits K, Fowler PJ, Birmingham TB and Litchfield RB. A case series of lateral opening wedge high tibial osteotomy for valgus malalignment. Knee Surg Sports Traumatol Arthrosc 2013; 21: 152-160.

[4] Brouwer RW, Jakma TS, Bierma-Zeinstra SM, Verhagen AP and Verhaar J. Osteotomy for treating knee osteoarthritis. Cochrane Database Syst Rev 2005; Cd004019.

[5] Nazem K, Fouladi A and Chinigarzadeh M. Double tibial osteotomy for bow leg patients: A case series. J Res Med Sci 2013; 18: 1092-1096.

[6] Miniaci A, Ballmer FT, Ballmer PM and Jakob RP. Proximal tibial osteotomy. A new fixation device. Clin Orthop Relat Res $1989 ; 250-259$.
[7] Fujisawa Y, Masuhara K and Shiomi S. The effect of high tibial osteotomy on osteoarthritis of the knee. An arthroscopic study of 54 knee joints. Orthop Clin North Am 1979; 10: 585-608.

[8] Kumagai K, Akamatsu Y, Kobayashi H, Kusayama Y and Saito T. Mosaic Osteochondral Autograft Transplantation Versus Bone Marrow Stimulation Technique as a Concomitant Procedure With Opening-Wedge High Tibial Osteotomy for Spontaneous Osteonecrosis of the Medial Femoral Condyle. Arthroscopy 2018; 34: 233-240.

[9] Chae DJ, Shetty GM, Wang KH, Montalban AS, Jr., Kim JI and Nha KW. Early complications of medial opening wedge high tibial osteotomy using autologous tricortical iliac bone graft and T-plate fixation. Knee 2011; 18: 278-284.

[10] Kuremsky MA, Schaller TM, Hall CC, Roehr BA and Masonis JL. Comparison of autograft vs allograft in opening-wedge high tibial osteotomy. J Arthroplasty 2010; 25: 951-957.

[11] Han SB, Lee DH, Shetty GM, Chae DJ, Song JG and Nha KW. A "safe zone" in medial open-wedge high tibia osteotomy to prevent lateral cortex fracture. Knee Surg Sports Traumatol Arthrosc 2013; 21: 90-95.

[12] Hooper NM, Schouten R and Hooper GJ. The outcome of bone substitute wedges in medial opening high tibial osteotomy. Open Orthop J 2013; 7: 373-377.

[13] Nawas HT, Vansadia DV, Heltsley JR, Suri M, Montgomery S and Jones DG. Factors Affecting the Union of Opening Wedge High Tibial Osteotomy Using a Titanium Wedge Plate. Ochsner J 2016; 16: 464-470.

[14] Hantes ME, Natsaridis P, Koutalos AA, Ono Y, Doxariotis N and Malizos KN. Satisfactory functional and radiological outcomes can be expected in young patients under 45 years old after open wedge high tibial osteotomy in a long-term follow-up. Knee Surg Sports Traumatol Arthrosc 2018; 26: 3199-3205.

[15] Villatte G, Erivan R, Fournier PL, Pereira B, Galvin M, Descamps $\mathrm{S}$ and Boisgard S. Opening-wedge high tibial osteotomy with a secure bone allograft (Osteopure) and locked plate fixation: Retrospective clinical and radiological evaluation of 69 knees after 7.5 years follow-up. Orthop Traumatol Surg Res 2015; 101: 953-957.

[16] Zorzi AR, da Silva HG, Muszkat C, Marques LC, Cliquet A, Jr. and de Miranda JB. Opening-wedge high tibial osteotomy with and without bone graft. Artif Organs 2011; 35: 301-307.

[17] Jacobi M, Villa V, Reischl N, Demey G, Goy D, Neyret P, Gautier E and Magnussen RA. Factors influencing posterior tibial slope and tibial rotation in opening wedge high tibial osteotomy. Knee Surg Sports Traumatol Arthrosc 2015; 23: 2762-2768.

[18] Darees M, Putman S, Brosset T, Roumazeille T, Pasquier G and Migaud H. Opening-wedge high tibial osteotomy performed with locking plate fixation (TomoFix) and early weight-bearing but without filling the defect. A concise follow-up note of 48 cases at 10 years' follow-up. Orthop Traumatol Surg Res 2018; 104: $477-480$

[19] Lansdaal JR, Mouton T, Wascher DC, Demey G, Lustig S, Neyret $\mathrm{P}$ and Servien E. Early weight bearing versus delayed weight bearing in medial opening wedge high tibial osteotomy: a randomized controlled trial. Knee Surg Sports Traumatol Arthrosc 2017; 25: 3670-3678. 
[20] Giuseffi SA, Replogle WH and Shelton WR. Opening-Wedge High Tibial Osteotomy: Review of 100 Consecutive Cases. Arthroscopy 2015; 31: 2128-2137.

[21] Siboni R, Beaufils P, Boisrenoult P, Steltzlen C and Pujol N.
Opening-wedge high tibial osteotomy without bone grafting in severe varus osteoarthritic knee. Rate and risk factors of non-union in 41 cases. Orthop Traumatol Surg Res 2018; 104: 473-476. 